\title{
COMMUNICATEURS CULTURELS : CONFLITS ET PROCESSUS DE LÉGITIMATION
}

\section{Lenay Alexandra Blason ${ }^{1}$}

À l'heure actuelle, la plupart des organisations culturelles ne peuvent plus se passer de faire la preuve de leur efficacité et de la satisfaction du public, tout en garantissant de la visibilité, autrement dit, tout en communiquant explicitement avec les parties concernées. Après les années que l'on appelle «de la croissance», les fonds alloués par l'Etat aux activités culturelles n'ont cessé de diminuer ou, dans le meilleur des cas, la décentralisation a donné lieu à une plus grande participation des collectivités locales dans la gestion financière de ce type d'organisations.

Dans ce paysage, on a confié à un nouveau métier la tâche de garantir la survie et de donner un nouvel élan au vieux théâtre, à l'ancien musée d'histoire naturelle, au parc botanique, au centre culturel de la ville. Dans un essai de généralisation, nous allons lui assigner un nom «commun»: le chargé de communication ${ }^{2}$, autrement dit celui qui

1 Doctorante au Département de Communication UCL. Membre du Laboratoire d'Analyse de Systèmes de Communication des Organisations (LASCO).

2 Dépendant du type d'organisation, cette fonction est dénommée «promoteur culturel», «animateur», «chargé de relations publiques», «conservateur», «responsable de ventes», ou encore, «communicateur».

Recherches en communication, $\mathrm{n}^{\circ} 25$ (2006). 
a la responsabilité de mettre au point la stratégie de communication et, souvent, de prendre la parole au nom de l'organisation.

Dans cet article, nous allons travailler dans une double perspective. D'abord, nous nous mettrons dans la peau d'un traducteur, en essayant de résumer et de commenter les interventions des chargés de la communication de différentes organisations culturelles en Belgique ${ }^{1}$. Quel sens donnent-ils à leur pratique professionnelle ? Quelles sont les tâches pour lesquelles ils ont été embauchés? Quels sont leurs défis, leurs satisfactions, leurs frustrations?

Dans la deuxième partie, nous tenterons de relever les convergences et les différences entre les points de vue, dans l'intention d'identifier les modes de légitimité et les enjeux de légitimation qui se dégagent de leurs discours. Quels sont les conflits de légitimité qui émergent de leur interaction avec les parties concernées? Quels aspects de leur «savoir-faire» constituent des atouts pour la recherche d'un espace légitime au sein des organisations culturelles? Est-ce que les communicateurs culturels ressentent un manque de légitimité ? Mais, avant d'aller plus loin, il faut faire le point sur ce que l'on entend dans cet article par «organisation culturelle».

\section{Les organisations culturelles : de quoi parle-t-on ?}

Le mot «culturel» suffit-il pour souligner la spécificité d'un type d'organisation? En effet, cet adjectif fait référence à tout ce qui est «relatif à la culture d'une société ou d'un individu, à son développement» ou «qui vise à répandre certaines formes de culture ${ }^{2}$. Donc, les organisations culturelles sont a priori des espaces où en même temps on stocke, on produit et l'on diffuse la «culture». Mais la définition de «culture» est elle-même assez diffuse et difficile à saisir.

En nous penchant sur l'approche fonctionnaliste de Malinowski', à notre avis une des plus pertinentes et complètes, la culture est un appareil instrumental qui permet à l'homme de mieux résoudre les problèmes concrets et spécifiques qu'il doit affronter dans sa relation

1 Ces interventions ont été faites lors de la conférence «Travailler dans le secteur culturel», le 17 février 2005 à l'UCL.

2 Le petit Larousse. Paris, Ed. Larousse, 2003, 289.

3 Malinowski, B. Une théorie scientifique de la culture et autres essais. Paris, Maspero, 1980. 
avec son milieu social et naturel. Ainsi, la culture comprend aussi bien le système de connaissances et les outils matériels permettant notre relation avec la nature, que les très diverses façons d'interagir avec les autres, en commençant par le langage.

De cette définition, une chose est évidente : quand nous parlons d' «organisation culturelle», nous faisons référence à un éventail très large et hétérogène d'organisations. Néanmoins, le terme ne va pas plus loin dans la caractérisation du fonctionnement de ces formes sociales. Rien n'a été avancé à propos de leur structure, leurs acteurs, leurs moyens de financement. C'est pourquoi il est nécessaire d'expliciter le cadre précis de cette réflexion, en nous en tenant à la matière primaire de notre travail -les interventions d'un groupe de communicateurs agissant dans le secteur culturel belge.

C'est ainsi qu'avec le terme «organisation culturelle» nous désignerons un type d'organisation dont la spécificité est la création, la diffusion ou la distribution de biens et de services culturels, mais qui est fort institutionnalisée, reconnue en tant que telle et non-lucrative à titre principal. Dans la plupart des cas, il s'agit d'activités financées par l'Etat ou qui dépendent fortement de lui, ce qui permet de les rapprocher du dénommé «secteur public». Il s'agit encore d'un nombre important d'organisations, mais qui n'y inclût pas, d'abord les grandes entreprises culturelles dont le fonctionnement ressemble beaucoup à celui de n'importe quelle autre entreprise du secteur privé, et puis les petits groupes pas très formalisés, que l'on appelle souvent «culture off» ou «culture underground».

Quelle est alors la spécificité de ces organisations? Peut-on les assimiler à des organisations du secteur public ou du secteur associatif? Quels types de changements ont-elles vécus dans les dernières années? Quels sont leurs nouveaux défis?

\section{Une typologie pour les institutions culturelles}

En dépassant l'adjectif «culturel» qui nous conduit à penser d'un côté à l'activité (ou produit) et de l'autre au statut, les organisations culturelles ont quelques traits typiques de l'ensemble des formes que prend l'action organisée.

D'abord il s'agit d'institutions qui jouent un rôle de médiation. Cette caractéristique a été largement étudiée par les différentes théories 
sociologiques de la médiation ${ }^{1}$, qui ont essayé d'identifier tout ce qui s'interpose entre l'œuvre et la réception, entre l'œuvre et le public. Par ailleurs, d'autres théories sociologiques, telle que la théorie des champs ${ }^{2}$ et la théorie de la reconnaissance ${ }^{3}$, ont essayé elles aussi de décodifier les structures sous-jacentes (type de capital, hiérarchies, rapport de domination) dans la production artistique, sa diffusion et sa réception.

L'œuvre du sociologue interactionniste Howard Becker ${ }^{4}$ (Les mondes de l'art, 1982) explore, à partir d'une description des actions et interactions sociales, la fonction de l'institution culturelle dans le monde de l'art. Il reprend dans sa démarche l'idée de réseau, une idée qui lui permet de concevoir l'art comme une activité collective où participent aussi bien les artistes que beaucoup d'autres instances médiatrices : les personnes ${ }^{5}$, les professions ${ }^{6}$, les institutions ${ }^{7}$, les identités collectives ${ }^{8}$ parmi d'autres 9 .

L'idée de réseau est, néanmoins, étroitement liée à la communication. Rappelons les travaux des sociologues de la traduction, M. Callon et B. Latour ${ }^{10}$, qui ont envisagé l'organisation comme un ensemble d'entités enchaînées, humaines ou non humaines, qui sont définies par leurs

1 Hennion, A. La Passion musicale : une sociologie de la médiation. Paris, Métailié, 1993./ Heinich, N. La sociologie de l'art. Paris, La Découverte, Collection Repères, 2004.

2 Bourdieu, P. Les Règles de l'art. Genèse et structure du champ littéraire. Paris, Seuil, 1992. / Bourdieu, P. La distinction : critique social de jugement. Paris, Editions de Minuit, 1979. / Bourdieu, P. «Les trois états du capital culturel»In : Actes de la recherche en Sciences Sociales, Vol 31, 2-3, 1979.

3 Bowness, A. The Conditions of success. How the Modern Artist rises to Fame. London, Thames and Hudson, 1989

4 Becker, H. Les mondes de l'art. Paris, Flammarion, 2006. / Becker, H. Symbolic interaction and cultural studies. Chicago, Chicago University Press, 1999.

5 Moulin R. L'artiste, l'institution et le marché. Paris, Flammarion, 1992.

6 Menger, P-M. La paradoxe du musicien. Paris, Flammarion, Champs, 1992.

7 Martorella, R. The Sociology of Opera. New York, Praeger Publisher, 1982.

8 Di Maggio, P. Nonprofits entreprises in the Arts : Studies in Missions and Constraint. New York, Oxford University Press, 1986.

9 Gomblaut A., Keravel A. «Les réseaux des mondes de l'art : un regard stratégique ». Table ronde à l'occasion de la XVème Conférence International de Management Stratégique, Annecy, Gèneve, 13-16 juin 2006.

10 Callon, M. La Science et ses réseaux. Genèse et circulation des faits scientifiques. Paris, La Découverte, 1991. / Callon, M. et Latour, B. La Science telle qu'elle se fait. Paris, EHESS, 1991. / Callon, M. et Latour, B. «Réseaux technico-économiques et irréversibilités» In Boyer, R. Figures de l'irréversibilité en économie. Paris, EHESS, 1991. 
rôles, leur programme, leur identité. Dans ce schéma, le traducteur -c'est-à-dire cette entité ou personne qui a la responsabilité de reconstituer le réseau, de le nommer, de l'analyser, de garantir la convergence des parties- a un rôle tout à fait essentiel pour le soutien du réseau.

C'est ainsi que, en assimilant d'emblée l'institution culturelle à une entité médiatrice agissant dans un réseau qui dépasse les frontières de l'organisation formelle, nous voulons souligner le rôle clé du chargé de la communication (ou de son équivalent), tout en nous inscrivant dans une démarche proche de celle de Menger du fait de nous intéresser aux professionnels de l'art et la culture.

Mais, au-delà de ces caractéristiques qui peuvent être attribuées à n'importe quelle organisation, privée ou publique, intervenant dans le «monde de l'art», une série d'autres critères permettant également de caractériser plus ponctuellement les institutions visées par cet article. Nous allons donc nous appuyer sur les travaux des chercheurs du Centre de Gestion Scientifique de l'Ecole des Mines de Paris (CGC) qui, en se penchant sur un certain nombre d'institutions culturelles en France ${ }^{1}$ dont le profil est proche de celles que nous prenons en compte dans cette réflexion, ont essayé de créer une typologie des organisations, «fondée sur les règles de leur 'physiologie'»".

Ces auteurs ont articulé les caractéristiques plus ou moins homogènes des institutions culturelles autour de trois axes :

- les missions et la conception

- les modes de gestion et de financement

- les enjeux des professions et de la professionnalisation

D'abord, et en règle générale, il s'agit d'organisations aux missions multiples et ambitieuses, pas toujours claires ou explicites, étant donné qu'elles sont nées de grands projets où l'opinion publique ou l'Etat jouent un rôle essentiel. Le caractère «politique» des missions officiellement attribuées par leur tutelle, très souvent lors de longs débats dans des instances publiques -parfois leur naissance s'appuie sur des décrets d'Etat- conditionne une certaine ambiguïté qui peut aussi se traduire

1 Leur travail prend comme point de départ l'intervention dans le Centre Pompidou, la Cité des Sciences et de l'Industrie, la Cité de la Musique, le Service d'Archive du Film et l'Institut du Monde Arabe.

2 Fixari D., Kletz F., Pallez F. «La gestion des institutions culturelles : est-elle spécifique? » In Cahiers de recherche, du Centre de Gestion Scientifique de l'Ecole des Mines (CGC), No. 11, Mars 1996. 
dans une plus grande marge de manœuvre à l'heure d'exécuter et planifier les fonctions.

\begin{abstract}
Une fois l'établissement (l'institution muséale) inauguré, ouvert pour l'éternité, son fonctionnement impose de jongler avec la pénurie de moyens, la pression des élus, du conseil d'administration, l'autorité des notables : si bien qu'en l'absence d'un projet collectif et fort, l'équipe professionnelle engagée dans cette aventure a souvent l'occasion de se perdre et de perdre de vue la finalité de son travail et à fonctionner au mieux sur un consensus 'mou'. Chacun pressent quelles sont les finalités et destinées de l'institution, mais en perd le fil, a du mal à l'exprimer et à en discuter. À la longue, après des années de fonctionnement, ou même dans le cas de la création, dès que les difficultés surgissent, chacun aura tendance à se replier sur ses attributions, en invoquant ses déceptions, en ressassant son amertume pour se désinvestir et désolidariser du projet collectif. ${ }^{1}$
\end{abstract}

Les missions plus ou moins typées vont de l'appui à la création artistique et la production culturelle («éveiller à l'art, à la culture») jusqu'à la promotion des différentes expressions de l'art ou la préservation du patrimoine naturel ou culturel d'une région, au travers d'activités d'animation et d'enseignement («former, enseigner»).

En outre, de grandes contradictions peuvent sortir d'une analyse plus exhaustive de ces missions : on leur demande des tâches ambitieuses avec des budgets limités; on leur demande à la fois d'attirer le grand public et de promouvoir l'œuvre des artistes d'avant-garde; on leur demande des réponses urgentes sur des questions qui s'inscrivent dans la longue durée des «phénomènes culturels».

Les mandats initiaux étant vagues et contradictoires, ils n'aident pas beaucoup à identifier ni les responsabilités, ni les moyens de réalisation et de gestion au quotidien. C'est ainsi que le «programme» devient l'expression formelle des fonctions et responsabilités souhaitées et que le moment de son élaboration devient le moment le plus important et critique dans la vie de l'organisation, permettant la coordination et l'explicitation des tâches pour les différentes parties.

1 Rasse P., Girault Y. «La démarche de projet dans les musées et les organisations culturelles » In Communication et organisation, No. 13, juillet 1998. 
Il semble utile de reprendre quelques questions posées par les chercheurs de l'école de Mines :

- Outre le flou peut-être inhérent à leur objet, les missions assignées à de telles institutions peuvent-elles être figées autrement qu'artificiellement, ne se construisent-elles pas sans cesse, au fur et à mesure de la naissance et du fonctionnement même du bâtiment?

- Qui a la charge de définir ces missions? La tutelle, être vague qui a en en principe la responsabilité, est-elle en la matière autre chose que le lieu où s'élaborent les compromis entre les futurs utilisateurs, les diverses institutions ou acteurs partie prenante? ${ }^{1}$

Le flou des mandats va permettre aux dirigeants de ces organisations une certaine liberté de modifier la stratégie à un moment donné, en rendant plus difficile le pilotage de leur évolution et en contraignant ou en élargissant la marge de manœuvre des acteurs. Nous sommes face à une «cacophonie» qui s'exprime dans des conflits de reconnaissance entre les acteurs, tel qu'on le verra plus tard.

Au-delà de son haut degré d'institutionnalisation et de reconnaissance publique, la deuxième caractéristique des institutions culturelles concernées par cette réflexion est leur caractère non-lucratif à titre principal. Elles fonctionnent donc essentiellement grâce à une dotation budgétaire de l'Etat ou des collectivités locales. Néanmoins, cette allocation est de plus en plus risquée car elle dépend, d'une part, des décisions politiques qui se prennent ailleurs -bien que la renommée personnelle des dirigeants et leur réseau de relations peuvent beaucoup influer- et, d'autre part, de la réputation que l'organisation elle-même a vis-à-vis de l'opinion publique et des publics. Cette réputation garantit aussi la participation financière de sponsors variés. Donc la formule est claire : plus d'affluence du public conditionne des financements plus importants et, en retour, une marge de manœuvre plus large.

La ressource que constitue le 'crédit' qu'acquiert l'institution culturelle, au sens où certains sociologues des sciences parlent de 'crédit' d'un laboratoire de recherche, est donc liée aux ressources financières qu'elle peut mobiliser auprès de ses

1 Fixari, Kletz, Pallez, Op. cit., 10. 
tutelles, du public et des sponsors, avec un effet de boucle de renforcement entre le 'crédit' et les ressources. ${ }^{1}$

C'est pourquoi les institutions culturelles sont forcées de mettre en œuvre continuellement une stratégie événementielle -festivals, foires, prix, grandes expositions temporaires, journées spéciales- qui complexifie d'autant plus la gestion interne. Au-delà d'une routine de fonctions (conservation, création, animation d'activités), elles doivent organiser plusieurs événements attirant l'attention du grand public et, surtout, garantissant une large couverture médiatique. La participation de personnes très réputées dans le monde de l'art à ces événements -lesquelles doivent être fortement rémunérées- et l'utilisation de ressources techniques très sophistiquées renforce ainsi le cercle de la réputation. De ce fait, et du déploiement de toutes sortes de publicity (brochures, spots, affiches, t-shirts, etc.), les événements sont de plus en plus chers et, en retour, ont de plus en plus besoin de l'accroissement des ressources extrabudgétaires pour financer une partie de leurs coûts.

Nous pouvons facilement imaginer que le développement de ces activités demande un grand investissement de ressources humaines -«capacité gestionnaire», chez les chercheurs du Centre de Gestion Scientifique de l'école de Mines. Encore une fois, il faut faire mention du moment de l'élaboration de la programmation. Le «planning» est d'autant plus compliqué qu'il faut prendre en compte de multiples contraintes : disponibilités des artistes ou des œuvres, les temps de montage et démontage, la programmation d'autres institutions agissant dans le même domaine, le temps pour faire la promotion de l'événement, mais surtout la coordination entre des acteurs très divers.

En effet, pour mener à bien leurs missions, les organisations culturelles doivent engager des «professionnels» ou des «métiers» de différents domaines, parmi lesquels des conflits de reconnaissance sont toujours à l'ordre de jour. D'abord, le personnel spécialisé -professionnels de l'art, scientifiques ou artistes- qui se ressentent du fait d'être jugés aussi bien par leurs pairs que par le public et les médias; ensuite, le personnel d'appui technique qui parfois considère «faire aussi de l'art»-sonoristes, graphistes, commissaires, etc.- et, finalement, le personnel administratif qui vit avec beaucoup de malaise le fait de s'in-

1 Fixari, Kletz, Pallez, Op. Cit., 13. 
vestir tellement dans un travail très mal reconnu et, parfois aussi, très mal rémunéré par rapport à ceux qui les entourent.

C'est ainsi que des logiques professionnelles tout à fait diverses doivent cohabiter dans le cadre des organisations culturelles, un contexte où, comme on l'a vu plus haut, les mandats ne sont pas complètement standardisés et rigides, mais plutôt confus et complexes. Un exemple : il est très difficile de définir des horaires précis pour des employés qui sont souvent amenés à travailler les soirs ou les week-ends pour l'organisation des événements ou l'accueil des visiteurs. En résumé, il s'agit d'activités peu formalisées, difficiles à quantifier, qui se traduisent par des systèmes de gestion organisée ouverts, avec peu de règles et de normes, et avec des espaces de liberté très larges. Mais, en revanche, cette liberté implique pour le personnel une grande difficulté à se positionner et à préciser son rôle :

[...] on assiste à un certain malaise chez les titulaires des postes, qui vivent de plus en plus mal le manque de cadrage de leur activité et qui réclament, au bout d'un certain temps, une clarification des rôles, s'accompagnant d'une demande de reconnaissance de leur métier, de leur identité, passant notamment par une codification sociale très claire. ${ }^{1}$

Finalement, si l'on parle des dynamiques d'évolution des organisations culturelles dans les dernières années -l'introduction d'une gestion par projet, événementielle et commerciale ou d'autofinancement-, il faut ajouter aussi l'émergence de nouveaux conflits entre les logiques professionnelles à l'œuvre dans le secteur culturel.

En effet, les besoins de nouvelles compétences pour faire face à de nouveaux modes de gestion ont conditionné l'apparition de nouveaux acteurs cherchant à se positionner et à revendiquer leur expertise face aux métiers préexistants.

De leur côté, les anciens acteurs n'assimilent pas toujours bien les changements mis en œuvre : par exemple, la commercialisation des supports de communication où l'œuvre d'art ou la connaissance scientifique est d'une certaine façon «vulgarisée»; ou la nécessité de concevoir, en marge du projet classique d'exposition ou de publication, les types de produit susceptibles d'être commercialisés. C'est ainsi que l'activité de conception se valorise d'autant plus qu'elle conçoit

1 Fixari, Kletz, Pallez, Op. Cit., 19. 
aussi la possibilité de récupérer les fonds dépensés, ou, dans un effet de boucle, de donner de la visibilité et de la réputation à l'institution. Une logique d'efficacité, de compétition et de confrontation -maîtrisée par des nouveaux acteurs- casse ainsi les logiques légitimées par des éléments de jugement différents.

En résumé, dans ce contexte particulièrement changeant et peu stable des activités culturelles, on peut constater de plus en plus l'exacerbation de logiques professionnelles :

\begin{abstract}
[...] d'une part, la réputation joue un rôle fondamental pour les acteurs aux métiers reconnus, aux statuts forts et l'évaluation se fait par rapport à des référents externes (les commissaires d'exposition ne se sentent réellement jugés que par leurs pairs); d'autre part, pour les nouveaux métiers, le besoin de reconnaissance est très fort, mais la difficulté à les juger 'bureaucratiquement' tout aussi grande. Ils cherchent alors à visibiliser leur action, à se positionner par rapport aux autres métiers et finalement à s'engager dans une démarche de professionnalisation (faire reconnaître leurs compétences, les normer, les rendre descriptibles, transférables, et valorisables à l'extérieur, les institutionnaliser en créant des associations supports ou des diplômes...). ${ }^{1}$
\end{abstract}

Devant ce paysage, le besoin de chercher des compromis et des dispositifs de coordination plus efficaces conditionne l'apparition d'autres acteurs chargés de créer le lien entre acteurs divers. Parmi les nouveaux recrutés, on trouve très souvent des professionnels de la communication avec un profil commercial -chargés de relations publiques, de marketing, de promotion- qui ne connaissent pas nécessairement le contexte original des organisations culturelles et dont la légitimité est mise en cause par les anciens acteurs.

Il s'agit en d'autres mots des conflits entre diverses formes de légitimité conditionnés par les différents rapports de pouvoir, symbolique et formel, des acteurs, et exacerbés par l'absence d'un projet collectif fort et clair. C'est pourquoi de nouvelles méthodes de gestion, particulièrement la gestion par projets, se mettent en œuvre à l'heure actuelle dans le secteur culturel. Mais nous considérons que cette sommaire caractérisation des institutions culturelles suffit pour nous plonger dans la deuxième partie de cet article : l'analyse des interventions des communicateurs culturels.

1 Fixari, Kletz, Pallez, Op. Cit., 22. 


\section{Le métier de la communication par les communicateurs}

À présent, et pour clarifier notre démarche, il faut rappeler que nous allons nous appuyer sur des interventions de communicateurs du secteur culturel dans l'intention de tirer la plus grande quantité d'information permettant de comprendre la nature des conflits de légitimité auxquels ils sont confrontés.

Tel que nous l'avons avancé plus haut, en affichant une position épistémologique pluraliste, nous allons dans les pages suivantes résumer et commenter les exposés des acteurs au moment où ils sont invités à donner un sens et une explication à leur vécu au travail. Pour rendre plus claire cette exposition, nous avons choisi de l'organiser autour de trois aspects liés à leur pratique professionnelle :

- les compétences requises lors de leur embauche

- les contenus assignés aux postes

- les satisfactions et les aspects négatifs ou frustrations.

\section{Un «savoir être» au lieu d'un «savoir-faire»}

À l'occasion d'interviews d'embauche, il y a toujours une série de compétences qui sont sollicitées pour réaliser une certaine activité. D'après les interventions des communicateurs culturels, ces compétences seraient plutôt :

- avoir une grande capacité d'adaptation

- être disposé à réaliser toutes sortes de tâches (être polyvalent)

- avoir une grande disponibilité horaire

- avoir de la passion pour le travail dans le secteur

- être fort ouvert

- aimer le contact avec les gens

- savoir travailler en équipe

- maîtriser le travail de rédaction

- être capable d'élaborer un projet

Une lecture sommaire nous permet d'affirmer que, sauf les deux derniers points (connaissances de rédaction et capacité de conception et coordination), les autres compétences ne sont pas nécessairement liées 
à un domaine scientifique ou professionnel -notons que les exposants sont licenciés en spécialités diverses : sciences biologiques, langues, communication ou histoire de l'art. C'est-à-dire qu'ils ne sont pas embauchés pour une qualité technique spécifique ou un savoir-faire, mais plutôt pour des qualités psychologiques ou une attitude face à la vie au travail ${ }^{1}$.

Cette description a d'ailleurs une ressemblance frappante avec les traits idéaux de l'homme de la «cité par projet», selon les travaux de Boltanski et Chiapello :

[...] en mettant l'accent sur la polyvalence, la flexibilité de l'emploi, l'aptitude à apprendre et à s'adapter à des nouvelles fonctions plutôt que sur la possession d'un métier et sur les qualifications acquises, mais aussi sur les qualités d'engagement, de communication, sur les qualités relationnelles, le néomanagement se tourne vers ce que l'on appelle de plus en plus souvent le 'savoir être' par opposition au 'savoir' ou 'savoir faire'. ${ }^{2}$

C'est ainsi que l'on pourrait considérer le métier de la communication comme un des nouveaux métiers apparus lors des changements vécus dans les organisations culturelles. Cela expliquerait pourquoi la légitimité que l'on attribue à l'avance aux communicateurs n'est pas une légitimité figée, liée à un pouvoir formel, à un status légal, à un corpus de connaissances ou, même pas, à un charisme donné; mais plutôt une légitimité en construction qui renvoie aux enjeux de continuité-changement des valeurs ou de traits identitaires de l'organisation. Ils sont conviés -en tant que porte-paroles ou 'traducteurs' (en termes de Callon et Latour)- à réinventer la mission organisationnelle, combler

1 Dans sa démarche concernant la communication organisationnelle au sein des entreprises, Stéphane Olivesi remarque aussi l'importance croissante accordée par le management, lors de nouveaux recrutements, aux manières d'être, de se comporter, de s'exprimer «[...] Les salariés doivent manifester un ensemble de dispositions et de qualités leur permettant de satisfaire de nouvelles exigences. Ces dispositions recouvrent les catégories des savoir-être. Elles supposent l'intériorisation de règles de comportement et de normes de sociabilités » (Olivesi, S. La communication au travail. Grenoble, Presses de l'Université de Grenoble, 2002, p. 116).

2 Boltanski, L. et Chiapello, E. Le nouvel esprit du capitalisme. Paris, Ed. Gallimard, 1996, p. 151. 
le vide idéologique, justifier la pratique -en garantissant la visibilitéd'une organisation en train de changer.

\section{«Un boulot qui s’apprend sur le tas»}

Par rapport au contenu assigné à leurs postes de travail, les différents témoins se sont accordés sur le fait qu'au début de leur engagement, ils n'ont pas reçu un mandat clair ou précis : «On a été engagé pour faire n'importe quoi», a affirmé un des intervenants, «avec mon contrat, c'était la première fois que la notion d'animatrice culturelle a été définie».

C'est ainsi que la propre indéfinition de la charge -animateur, responsable du service de promotion, chargé des relations publiquesest le premier symptôme d'un décalage entre les compétences requises et le travail à faire. Mais, s'agit-il du même contenu dans tous les cas? En gros, sauf quelques particularités, les cinq exposants partagent le même avis par rapport aux tâches qu'ils doivent réaliser :

- la conception et la mise en œuvre de campagnes de promotion

- la conception et la coordination de projets

- le maintien des relations personnalisées avec les différents publics et avec les médias

- le travail avec les sponsors dans la recherche de fonds et subsides

- la conception d'une politique cohérente d'image institutionnelle

- la conception et le maintien du site Web et la publication (bulletin, journal, etc.) de l'organisation

Nous pouvons observer qu'il s'agit d'un travail très spécialisé nécessitant des connaissances spécifiques et d'un savoir-faire systématisé. Néanmoins, le fait qu'il n'y ait pas une identification claire de mandats et que les personnes embauchées n'aient pas dans tous les cas les connaissances nécessaires pour réaliser ce type de travail -les compétences requises n'étant pas non plus précisées-, laisse une grande marge à l'improvisation et à la gestion au quotidien : «c'est un boulot qui s'apprend sur le tas».

En revanche, il est si difficile de piloter et d'évaluer le travail des communicateurs que leur régulation et leur contrôle se réalisent plutôt par les résultats. Et cela devient une arme à double tranchant parce que la légitimation du métier se joue dans chaque situation particulière, ne 
dépendant ni de la réputation conférée de l'extérieur, ni d'une légitimé préalablement affirmée. L'acteur doit à la fois clarifier et expliciter le besoin de l'organisation d'une fonction de «communication» et construire un savoir-faire qu'il ne trouve pas au début de sa gestion.

\section{Les bonheurs et les malheurs du monde de la culture}

«C'est vrai qu'il est très intéressant de travailler dans un secteur passionnant où il est possible d'être toujours en contact avec des personnes différentes et de connaître toujours de nouvelles choses». En effet, tous les intervenants ont assuré aimer leur travail -«autrement nous serions partis»- et s'investir avec beaucoup d'enthousiasme dans tous les projets et tous les événements qu'ils sont chargés de mener à bien. Néanmoins, dans leurs discours nous pouvons identifier d'autres situations conflictuelles par rapport à leur pratique professionnelle.

D'abord, ils ont manifesté le sentiment de «ne pas faire les choses en profondeur». Dans son intervention, la responsable du service de promotion d'un centre culturel affirme que la pression des événements ne permet pas de prendre le temps de la réflexion et de se remettre en question : «J'aimerais bien prendre le temps de planifier avec beaucoup d'anticipation la stratégie de communication et aussi d'évaluer les résultats, mais on est toujours pressée, parce qu'il y a toujours des activités».

Tel que l'on a vu plus haut, les communicateurs culturels sont évalués par des résultats à très courts termes, tandis qu'ils aimeraient plutôt agir sur le changement de conduites -éveiller à la perception des différentes manifestations artistiques, créer une conscience de l'importance d'une œuvre ou d'une région, changer les perceptions identitaires de leurs organisations, etc.-, ce qui est seulement possible dans la longue durée. De ce décalage entre les attentes et les possibilités réelles, il ressort une espèce de «frustration» du métier qui commence à être perceptible au bout de quelques années.

Cela est mis en évidence par ces autres témoignages. Paradoxalement, au sentiment d'urgence s'ajoute la perception que : «on travaille dans un secteur qui ne bouge pas trop, où tout est extrêmement long et difficile».

Dans la caractérisation des organisations culturelles, nous avions déjà remarqué qu'il s'agissait de systèmes d'action organisée, de réseaux très dépendants de l'environnement, où participent des acteurs affichant des formes de légitimité différentes. Le communicateur est 
dans ce contexte un nouvel acteur, un acteur émergent qui est souvent chargé de faire le lien et de gérer les modes de coopération conflictuelle qui traversent l'organisation et qui la remodèlent constamment.

L'exemple du théâtre permet de montrer les enjeux des acteurs concernés dans ce réseau : le communicateur est interpellé d'un côté par les «artistes» souhaitant avoir un maximum de promotion pour leur spectacle; les dirigeants et les sponsors sollicitant une politique de communication efficace (l'organisation doit justifier sa raison d'être) et des politiques de commercialisation amortissant une partie des coûts, et finalement, par le public et les médias demandant la plus grande quantité d'information et de satisfaction. Ils sont au centre de la boucle de la réputation et de la reconnaissance : plus d'affluence de public, grâce à un travail de promotion bien fait, plus de réputation de l'organisation et des artistes grâce en grande partie aux bonnes relations avec les médias et, en somme, plus de garanties de fonds pour de nouveaux événements, qui doivent être à leur tour bien encadrés et bien promus, et ainsi de suite.

Ce travail demande un investissement en énergie et en temps très grand qui, selon les exposés, est très mal récompensé. Il s'agit de postes parfois mal rémunérés demandant une grande flexibilité horaire.

\section{Conflits de légitimité et processus de légitimation}

À présent, en croisant les caractéristiques particulières des institutions culturelles et les conclusions des interventions des chargés de la communication, nous pensons disposer des éléments nécessaires pour esquisser un canevas des conflits entre les différentes formes de légitimité et les processus de légitimation qui émergent de leur pratique professionnelle.

Tout d'abord, nous voulons récupérer les notions de «conflits» et de «processus». Vu que dans le cas du métier de la communication, nous ne partons ni d'une légitimité figée, ni d'une légitimité opérée par la nomination explicite (la description du poste), il est à notre avis plus pertinent de relever les rapports sous-jacents de force et de pouvoir qui marquent l'action commune, ainsi que les processus de construction d'une identité du métier, indispensable pour sa légitimation au sein des institutions culturelles. 
En effet, le besoin de communiquer à tout prix est une nécessité relativement nouvelle pour ces organisations dépendant de fonds et de subsides publics de plus en plus risqués, et prises, à l'heure actuelle, dans la «noria» de la réputation et de la reconnaissance qui caractérise les réseaux de l'art et la culture, spécialement de ce que l'on appelle l'industrie culturelle ${ }^{1}$. C'est ainsi que les acteurs chargés de cette fonction de la communication -nouveaux embauchés ou anciens acteurs se dotant de nouvelles qualifications et fonctions-, peuvent être analysés comme des acteurs émergents, censés redonner un sens aux organisations en train de changer et de combler l'absence d'un projet organisationnel fondé sur une idéologie légitimée.

Néanmoins, ils sont confrontés à un paradoxe : ils doivent stabiliser les contenus de leur «fonction», apprendre et systématiser un «savoir-faire» et remarquer la valeur et l'importance de leur travail, face au sommet managérial et aux autres acteurs dont l'enjeu consiste à les évaluer et les juger en fonction de leurs qualités, leurs performances, leur «savoir-être $»^{2}$. C'est ainsi que nous pouvons conclure qu'ils sont porteurs d'une légitimité très fragile qui se joue au quotidien à partir de résultats à très courts termes. Il est facile d'imaginer que ce type de légitimité est supposé être toujours remise en cause par des acteurs portant des légitimités essentialistes. Ces conflits sont d'ailleurs exacerbés par le fait que le système d'action organisée dans le domaine de la culture dépasse largement les frontières de l'organisation formelle : nous parlons alors d'un réseau qui compte des acteurs très divers, en commençant par les publics et les médias jusqu'aux sponsors et responsables politiques.

En revanche, du manque d'un mandat clair et spécifique, de l'absence d'un type de régulation explicite et du décalage entre la percep-

1 Nous voyons aussi l'interférence au moins de deux «mondes», au sens de Boltanski et Thévenot : celui de la «cité civique», incarnant la volonté du «Bien Commun» et de la «cité industrielle», fondée sur l'efficacité. Il apparaît d'ailleurs que les conflits entre ces deux mondes peuvent être gérés en appelant à un autre modèle, celui de la «cité par projet», dont le communicateur devient une figure essentielle (Boltanski, L. et Thévenot, Y. De la justification : économies de la grandeur. Paris, Ed. Gallimard, 1991).

2 D'après Stéphane Olivesi : «Le flou de ces compétences (communicationnelles) conditionne le déploiement d'un jeu social au sein duquel les jugements respectifs des acteurs les uns envers les autres jouent un rôle de plus en plus important » (Olivesi, S. La communication au travail. Grenoble, Presses de l'Université de Grenoble, 2002, p. 119) 
tion des compétences requises et les résultats de leur travail, les chargés de communication peuvent tirer des enjeux de légitimation non négligeables. À l'heure actuelle, ils sont devenus des acteurs incontournables dans la vie des organisations culturelles. Du lointain «n'importe quoi» jusqu'à «l'homme-orchestre» d'aujourd'hui, le métier évolue et se construit, si bien qu'il s'agit d'une évolution perpétuelle demandant beaucoup d'énergie et d'investissement. Les communicateurs sont favorisés par les changements de l'environnement où agissent les institutions culturelles. Nous avons déjà parlé de la diminution des fonds et de la nécessité de chercher de nouveaux subsides, mais il faut aussi ajouter l'émergence et la généralisation des nouvelles technologies de l'information et de la communication (TIC). De la maîtrise des TIC, le métier acquiert une spécificité et une spécialisation qui pourraient le rendre porteur d'une légitimité liée au savoir. En effet, les organisations doivent obligatoirement être au fait des nouvelles technologies. Les réticences des anciens acteurs s'écroulent face aux potentialités des sites Web, bulletins électroniques, multimédias, spots télé, communautés virtuelles, etc. Par ailleurs, avec ces nouvelles technologies, les délais de résultats se réduisent considérablement et cela fait gagner de la visibilité au travail des communicateurs.

Dans ce même sens, il faut remarquer l'importance des retombées médiatiques dans la légitimation du métier. L'arme la plus précieuse pour les communicateurs, et peut-être le seul moyen objectif de juger son travail, sont les «revues de presse». Pour une grande part, leur réputation se juge par la quantité d'information parue dans les journaux (car il est un peu plus difficile d'archiver les informations audiovisuelles). Tout le monde souhaite se voir représenté dans les médias et pour cela on est presque obligé de passer par le responsable de communication, celui qui rédige les communiqués de presse, qui organise les conférences, qui connaît de très près les journalistes spécialisés, qui maîtrise les technologies, qui a un réseau de relations d'autant plus large.

En résumé, les communicateurs incarnent parfaitement la fonction de médiation des institutions culturelles. Ils sont convoqués pour donner des réponses aux besoins de reconnaissance et de légitimation des différents acteurs, ainsi que de l'organisation elle-même. En garantissant la visibilité et la reconnaissance des autres et en justifiant l'agir organisationnel, ils justifient d'une certaine façon leur propre pratique professionnelle et bâtissent leur propre légitimité. Est-ce que cet état de choses correspond à leurs aspirations? 
Rappelons-nous à nouveau la source des frustrations de nos intervenants. En effet, ils sont coincés entre l'immédiat des résultats demandés et la lenteur des phénomènes impliquant le changement des conduites; entre l'urgence d'une stratégie événementielle et leur volonté d'une stratégie de communication mieux réfléchie visant aussi la longue durée; entre les envies de professionnaliser leur travail et les contraintes de la réalité au quotidien. Ne sommes-nous pas, encore une fois, face aux paradoxes d'une utopie de la communication?

Le thème de la 'société de la communication' s'ajuste très bien à la fois à ce trait du politique et à la nécessité incontournable d'avoir à imaginer un mode d'organisation sociale qui permette de s'adapter au changement. Le refus du politique ne peut toutefois tenir lieu, à lui seul, de politique. ${ }^{1}$

A présent, la coïncidence du profil des communicateurs culturels avec celui de l'homme de la «cité par projets» de Boltanski et Chiapello acquiert toute sa signification. En effet, dans le secteur culturel comme dans tant d'autres secteurs -je pense notamment au secteur associatif-, les communicateurs constituent une nouvelle «figure» symbolisant et incarnant les contradictions d'un système d'action organisée qui a dû s'adapter aux changements idéologiques récents, dépouillant la culture et en général l'action commune de tout contenu politique ${ }^{2}$.

La question change alors de sens : en légitimant l'organisation et leurs acteurs, les communicateurs ne trahissent-ils pas souvent leur éthique professionnelle et leur compromis politique? Ou encore : sur quelles prémisses veulent-ils construire leur légitimité?

Néanmoins, nous doutons du fait qu'il s'agisse d'une question qui préoccupe a priori les communicateurs dans le secteur culturel. Si l'on s'accorde sur le constat médiologique affirmant que fabriquer une identité est un processus symbolique qui dépend de la création «[...] d'un milieu de transmission singulier autour d'un certain nombre de procédures de reconnaissance et d'interconnaissances (congrès, abrégés,

1 Breton, $\mathrm{Ph}$. L'utopie de la communication : le mythe du «village planétaire». Paris, La Découverte/Poche, 1997 (1 ${ }^{\text {ère }}$. Edition,1992), p. 95.

2 Dans un phénomène de «isomorphisme institutionnel ?» (Di Maggio, Op. Cit., 1986), les institutions culturelles ont fini par se ressembler de plus en plus à d'autres formes de l'action organisée. En accusant les modes de gestion propres des organisations du secteur public, elles sont forcées à rentrer dans la logique commerciale du secteur entrepreneurial. 
cellules, revues, journaux, centres de recherche, colloques, etc.)» ${ }^{1}$, l'existence d'une intention d'autolégitimation du métier n'est pas très évidente. En effet, à part des cas très isolés, attachés fondamentalement aux centres des recherches, il y a peu d'actions cherchant à expliciter et à donner des réponses aux problématiques liées à la pratique professionnelle des communicateurs culturels. La construction d'une identité (identité et légitimité étant étroitement liées) de ce métier, dont la dénomination même n'est pas toujours homogène, reste une tâche demandant une énergie et un temps qui manque aux professionnels ou qui accuse elle aussi, peut-être, la lenteur du secteur.

1 Debray, R. Manifestes médiologiques. Paris, Ed. Gallimard, 1994. 
